\title{
Effect of Surface Temperature on Adhesion of Nanoparticle-coated Microparticles
}

\author{
Chaitanya Krishna Prasad Vallabh ${ }^{a}$, James D. Stephens ${ }^{a}$, Grazyna Kmiecik-Lawrynowicz ${ }^{b}$, \\ Santokh Badesha ${ }^{b}$ and Cetin Cetinkaya ${ }^{a^{*}}$ \\ ${ }^{a}$ Photo-Acoustics Research Laboratory \\ Department of Mechanical and Aeronautical Engineering \\ Center for Advanced Materials Processing \\ Clarkson University \\ Potsdam, New York 13699-5725, USA \\ ${ }^{b}$ Xerox Corporation \\ Webster, New York 14580, USA
}

Version 02.12

May 13, 2016

* Author to whom correspondence should be addressed.

Email: cetin@clarkson.edu | Phone: (315) 268-6514 | Fax: (315) 268-6695 | 


\begin{abstract}
Toner microparticles are coated with silica nanoparticles for modulating their adhesiveness to optimize two competing requirements in the electrophotographic process: low adhesion for facilitating particle transport and high adhesion with deposited substrates prior to high temperature fusing. In addition to the adhesion effect of nanoparticle coating, nanoparticlecoated toner microparticles are subjected to various thermal fields during utilization. Understanding the effect of temperature on particle adhesion properties has the potential to improve energy efficiency, process predictability and print resolution. In current work, the effect of surface temperature on the adhesion bond between single nanoparticle-coated toner microparticles and a silicon substrate is investigated in a non-contact/non-invasive manner by monitoring the rocking dynamics of acoustically excited single microparticles on a silicon wafer surface. In the reported spectral experiments, at a range of substrate temperature levels from 15 to $30^{\circ} \mathrm{C}$, the work-of-adhesion values of the surface-particle bond are evaluated from the acquired rocking resonance frequencies of the single toner microparticles. Rayleigh Surface Acoustic Waves (SAW) are employed to generate highly controllable surface motion to excite the vibrational modes of deposited microparticles, through which the out-of-plane transient responses of their apexes are acquired using a laser vibrometer. In addition to surface temperature, the effect of coating nanoparticle spatial distribution on the adhesion properties of the microparticle is observed and explained by proposing possible contact configurations, depending upon how silica nanoparticles are accumulated in and around the particle-surface contact zone. Finally, it is observed and reported that the relative humidity $(\mathrm{RH})$ can play a significant role in microparticles rocking motion dynamics and adhesion.
\end{abstract}


Keywords: single microparticle adhesion, nanoparticle coated toner, toner adhesion modulation, work-of-adhesion of toner, non-contact ultrasonic technique, surface acoustic waves. 


\section{Introduction}

In a wide spectrum of industrial processes and natural phenomena, both microparticle adhesion force prediction and its statistical variation are critically essential for process optimization. In recent years, precise control over the microparticle size and shape distributions has been achieved using production techniques for electrophotography (e.g. Emulsion Aggregation (EA) technology [1]) and additive manufacturing (e.g. plasma spheroidization). Therefore, understanding the effects of adhesion force distribution on the surface of an individual microparticle has become more relevant for developing processes to obtain narrower property distributions, leading to improved deposition performance. In electrophotographic printing/copying, electrostatic forces are utilized to move and transfer charged toner particles from a reservoir to a surface for the purpose of producing high-quality images, so accurate adhesion characterization is essential for process optimization. Various microparticle adhesion methods which primarily employ centrifugal, electric field detachment, aerodynamic (air gun), hydrodynamic, pendulum impact-separation, and ultrasonic vibrations techniques have been reported for microparticles [2]. Due mainly to limited accuracy and spatial resolution of adhesion methods, disagreements over the relative importance of electrostatic and non-electrostatic contributions to toner adhesion have been reported [3]. For instance, the results of Hays et al. [3, 4] indicate that toner adhesion is dominated by electrostatic forces, while the analyses of Rimai et al. [5] appear to show that toner adhesion at micro-scale is mainly as a result of van der Waals forces. Some investigations such as those of Gady et al. [6] suggest that both van der Waals and electrostatic forces can play significant roles in microparticle adhesion. In addition to contributors of adhesion force, our recent experimental results [7, 8] indicate that their spatial distribution over the surface of the individual particle must be understood for more precise 
prediction of production control parameters as the microparticle size and geometry has now become better controlled. However, the temperature dependency of adhesion in microparticles attracted rather limited attention, even though many processes expose microparticles to large temperature variations.

Numerous simulation-based computational studies have been conducted to predict the temperature effect on adhesion energy. The surface energy dependence on the surface temperature, for example, was computationally estimated for solid nickel [9] for the surface temperature range $27-1327^{\circ} \mathrm{C}(300-1600 \mathrm{~K})$ using molecular dynamics (MD) simulations and for copper using Monte Carlo simulations in the broad range of $-273-927^{\circ} \mathrm{C}(0-1200 \mathrm{~K})$ [10]. Changes in the surface energies of amorphous carbon films were reported for the relatively narrow temperature range of $20-95^{\circ} \mathrm{C}$ (293-368 K) employing a contact angle-based experimental method [11]. The effect of temperature on the surface energy of diamond-like carbon and tetrahedral amorphous carbon (ta-C) coatings considered as a protective layer for biological implants owing to their superior biocompatibility [12] were also investigated and reported [11].

The focus of current work is to understand and evaluate the surface temperature effects on nanoparticle coated toner microparticle adhesion. Progress in the area could lead to energy efficient optimized processes and increased process predictability as well as high-resolution images. In the reported study, based on a non-contact measurement approach, the surface energy dependence of the adhesion bond between a single nanoparticle-coated toner microparticle and a silicon substrate on the substrate surface temperature is quantified. Emulsion Aggregation (EA) 
nanoparticle-coated toner microparticles are utilized in the experiments for studying the effect of surface temperature on their adhesion properties. EA is a chemical process used to grow nearperfect spherical microparticles with narrow diameter distributions from sub-micron sized precursors. EA toner particles are often coated with nanoparticles in order to modulate their adhesiveness as bare particles have high adhesion energy compared to milled microparticles and surface area coated toner particles [13]. High surface energy of bare toner particles make the particle transport process in printing/copying machines a challenge, therefore understanding the properties of coated toner particles is important for process optimization. In reported experiments, the decrease in the surface energy of the substrate (silicon) with the increase in surface temperature is investigated by studying the work-of-adhesion of microparticles for a temperature range of $15-30^{\circ} \mathrm{C}$. The microparticle-substrate adhesion is evaluated using a noncontact/non-invasive approach based on ultrasonic excitation and interferometric motion sensing techniques similar to those reported in [14]. Experimental data demonstrating the effect of surface temperature on the work-of-adhesion of the microparticle-substrate interface is presented and discussed.

The non-contact Surface Acoustic Wave (SAW) based ultrasonic approach reported in this work can be applicable to various other applications dealing with microparticles and temperature variations, such as biological cells (e.g., platelets, red blood cells) [15] and the coatings of biological implants [12] as well as toner particle adhesion in copying/printing applications [14, $16]$. 


\section{Experimental Approach and Materials}

The key objective of the reported experimental study is to determine the effect of surface temperature on the work-of-adhesion between nanoparticle-coated microparticles (toner) and substrates in a non-contact/non-invasive repeatable manner. Experiments were designed and performed to evaluate the effect of surface temperature on the dynamic and adhesion behavior of single toner microparticles fully covered with silica nanoparticles. The toner microparticles are deposited on a silicon substrate affixed on top of a stack of Peltier thermoelectric coolers (Fig. 1). Deposited microparticles were acoustically base-excited by a Rayleigh Surface Acoustic Wave (SAW) field driven by a commercial ultrasonic transducer with a central frequency of 1.0 MHz (A103R, Panametrics, Waltham, Massachusetts, USA) affixed to an acoustic wedge for a range of substrate surface temperature levels $\left(T_{\mathrm{s}}\right)$ (Fig. 1). It was previously reported that the temperature of the substrate decreases the surface energy of the substrate [9-11, 17] causing the change in particle-substrate bond stiffness, thereby affecting the adhesion of toner microparticles.

\subsection{Materials}

A set of 23 emulsion aggregation toner microparticles coated with silica nanoparticles, identified as Sample Set 01 (SS01), are utilized in the experiments performed (Fig. 2). The mean diameter of the microparticles in the sample set is approximately $15 \mu \mathrm{m}$ (Table 1), with a mass density of $1000 \mathrm{~kg} / \mathrm{m}^{3}$. The outer layer of the toner microparticles, provided by Xerox Corporation (Webster, Rochester, New York, USA), comprise a polymeric resin coated with $40 \mathrm{~nm}$ (mean diameter) silica nanoparticle additives encompassing the surface of the toner particle (i.e. Surface Area Coverage (SAC) of $100 \%$ ) to modulate the adhesiveness of the toner. The scanning electron 
microcopy (SEM) image showing the surface morphology of the 100\% SAC toner microparticles is depicted in Fig. 2 (a) [13]. The optical microscope image of a particle employed in the experiments is included in Fig. 2 (b), the shapes and sizes (diameter) of the microparticles are determined and validated with such images. The optical images were captured employing an optical microscope (BXFM-ILHSPU, Olympus, Tokyo, Japan) unit integrated with the microscopic adapter (OFV 074, Polytec, Waldbronn, Germany) and a charge-coupled device (CCD) camera (A102f, Basler Inc., Exton, Pennsylvania, USA).

\subsection{Experimental Set-up}

The instrumentation diagram of the current SAW-induced microparticle rocking and rolling experimental set-up is depicted in Fig. 1. The instruments employed in the experimental setup include (i) a pulser/receiver unit (5077PR, Panametrics, Waltham, MA), (ii) a commercial ultrasonic transducer with a central frequency of $1 \mathrm{MHz}$, (iii) a digitizing oscilloscope (TDS 3052, Tektronix, Beaverton, Oregon, USA) with a frequency bandwidth of $500 \mathrm{MHz}$, (iv) a laser fiber interferometer (OFV511, LDV, Polytec, Waldbronn, Germany) and (v) a vibrometer controller (OFV3001, Polytec, Waldbronn, Germany). The non-contact ultrasonic experimental setup is developed to study the particle vibrational dynamics under an applied substrate surface temperature $\left(T_{s}\right)$. The vibrational response of a microparticle is related to the adhesion properties of the particle-substrate contact zone. The experimental setup detects the transient out-of-plane displacement response of the particle excited by an ultrasonic transducer in the temporal domain under varying levels of substrate temperature $\left(T_{s}\right)\left(15-30^{\circ} \mathrm{C}\right)$. The surface temperature $\left(T_{s}\right)$ on silicon substrate was controlled by varying the current input using a power supply unit (1760A, BK Precision, Yorba Linda, California, USA) to the Peltier thermoelectric cooling assembly 
(TEC1 - 12706, Hebei IT, Pudong, Shangai, China). The surface temperature on the silicon substrate was validated and recorded by a contact digital thermometer utilizing a small form ktype thermocouple (Dual Channel Digital Thermometer, Signstek, Shenzhen, Guangdong, China) and verified by a non-contact infrared (IR) thermometer (ST350, AGPtEk, Brooklyn, New York, USA). The experimental setup is based on a previously developed non-invasive ultrasonic excitation and laser interferometry sensing technique detailed in $[14,16]$. Microparticles are dry-deposited on the substrate. During each experimental cycle, the ultrasonic transducer periodically emits an acoustic field by converting an electric signal (square pulse) originating from the pulser/receiver unit into a transient pressure (longitudinal) strain field. This strain field is transformed into a surface-localized Rayleigh SAW by passing through the acoustic wedge made of Rexolite ${ }^{\circledR}$ at a particular angle of approach $\left(\theta_{\mathrm{w}}=42.2^{\circ}\right)$, thereby exciting the silicon substrate with a surface bound wave pulse [17]. The pulse repetition frequency (PRF) is set at $100 \mathrm{~Hz}$ on the pulser/receiver and the pulser voltage to $400 \mathrm{~V}$. The transient out-of-plane displacement response of the particle is acquired through the laser fiber interferometer and the vibrometer controller setup. The acquired out-of-plane displacement response is digitized, averaged and recorded employing a digitizing oscilloscope. In the reported experiments, the digital sampling frequency of the oscilloscope is 5 Giga samples per second, with each sample comprising an 8-bit encoded amplitude measurement. Each waveform utilizes an averaging of 512 waveforms to maximize the signal to noise ratio in the acquired response.

The local relative humidity $(\mathrm{RH})$ in the vicinity of the experimental setup is controlled using a dehumidifier (FDR25S1, Frigidaire, Charlotte, North Carolina, USA). The dehumidifier was integrated into the experimental setup in order to maintain consistent $\mathrm{RH}$ and to acquire 
repeatable data. The experimental zone is enclosed with a transparent plastic film bag, referred as the experimental enclosure here. The dry air was introduced into the experimental enclosure through a duct (including an air filter and fan driving the forced air) made using tin foiled pipe of 6" diameter and an intake window $(6 \times 10$ in) placed on top of the exhaust vent of the dehumidifier.

\subsection{Experimental Procedure}

For systematically evaluating the change in the adhesive properties of toner microparticles, an experimental procedure was developed based on the changes in their rocking resonance frequencies for four temperature levels (i.e. $15,20,25$, and $30^{\circ} \mathrm{C}$ ). The primary utility of the current experimental procedure is to acoustically excite the microparticles deposited on the silicon wafer substrate and observe/record their dynamic responses with aid of a laser interferometer for varying levels of substrate temperature $\left(T_{\mathrm{s}}\right)$. New and unused silicon substrates were used in the experiments. Prior to the experiments the substrates were rinsed with deionized (DI) water. Then the microparticles (toner) are dry-deposited on the silicon substrate and stored in a petri dish with desiccant (silica gel) to dry out any moisture on the substrate. As depicted in the schematics of the experimental setup (Fig. 1), the silicon substrate (with the deposited particles) bonded with the acoustic wedge assembly is placed on top of the stacked Peltier coolers for experimental data acquisition.

Dry air is continuously introduced into the experimental enclosure (approximately 10 inches above the substrate-particle setup) to lower the local humidity, to reduce the chances of capillary condensation and/or liquid bridge formation. The silicon substrate assembly with the acoustic 
wedge, transducer and the deposited toner microparticles are placed under the objective $(5 \times)$ of the microscope (Fig. 2(c)). The substrate surface temperatures were cycled from 15 to $30^{\circ} \mathrm{C}$ upscale and downscale three times, to relax the particles and break secondary loose bonds. Following the relaxation cycles, a set of isolated single microparticles are located and mapped for data acquisition. By employing an objective lens of 50× magnification a single microparticle is focused, the vibrometer probe laser spot of approximately $1 \mu \mathrm{m}$ is then directed on to the apex of the microparticle surface. The temporal responses of the mapped microparticles and the silicon substrate (Fig. 3) are acquired at surface temperatures of 15, 20, 25 and $30^{\circ} \mathrm{C}$ employing the oscilloscope. These transient out-of-plane displacement (in nanometers) responses of the microparticles and the substrate are a result of the SAW excitation, excited with an ultrasonic transducer of $1 \mathrm{MHz}$ central frequency and a pulser voltage of $400 \mathrm{~V}$ as detailed above. The displacement responses of the particles and the substrate are processed into their respective spectral domain using a Fast Fourier Transform (FFT) routine (Fig. 3). From the spectral responses, the rocking resonance frequency of each particle is extracted and based on a previous adhesion model [16], the work-of-adhesion values are then calculated. The obtained work-ofadhesion is related to the surface energy and compared with van der Waals-Guggenheim equation for theoretical validation of the experimental results. The temporal responses for the particles were also obtained in a reverse order (i.e., $30,25,20$ and $15^{\circ} \mathrm{C}$ ) in order to observe any temperature order effects and maximize the repeatability of the experimental procedure. It was observed that data acquisition based on the surface temperature order had no effect on the values of acquired rocking resonance frequencies, thus indicating that the reported procedure is repeatable. The current data set was acquired in ambient conditions, namely, at a room temperature of $23^{\circ} \mathrm{C}$ and experimental enclosure relative humidity $(\mathrm{RH})$ of $25 \%$, and $\mathrm{RH}$ of 
$14.5 \%$ (for SS01_MR16). During experiments, the room temperature and RH was observed and recorded using a hand-held thermohygrometer (EW-17030-20, Cole-Parmer, Vernon Hills, Illinois, USA). Capillary condensation is not expected at either $\mathrm{RH}=25 \%$ or $14.5 \%$ for the temperature range of $15-30^{\circ} \mathrm{C}$, as it is reported that capillary condensation () occurs at approximate $\mathrm{RH}$ values of $60-85 \%$ for the reported temperature range. [18]

\section{Experimental Data and Results}

Employing the experimental procedure detailed above, the temporal responses for each particle in the particle sample set SS01 consisting of 23 microparticles were acquired and processed into its spectral domain (Fig. 3) to determine its rocking resonance frequency at four surface temperature levels $\left(15,20,25\right.$ and $\left.30^{\circ} \mathrm{C}\right)$. A particle makes an in-plane (lateral) damped rocking motion at its rocking resonance frequency. The data was processed by employing a band-pass Infinite Impulse Response (IIR) filter with a lower cut-off frequency of $35 \mathrm{kHz}$ and an upper cutoff frequency of $200 \mathrm{kHz}$ for eliminating noise level and frequency components well above the expected range of rocking resonance frequency. For the current sample set, based on the previously reported values for similar microparticles [7, 8], the expected rocking resonance frequency is estimated to be $35-160 \mathrm{kHz}$. The acquired rocking resonance frequencies $f_{\mathrm{R}}$ of the microparticles in the sample set and corresponding work-of-adhesion $\left(W_{\mathrm{A}}\right)$ values of the microparticles for varying surface temperatures are reported in Table 1 along with the percentage reduction of $W_{\mathrm{A}}$ values from surface temperature of 15 to $30^{\circ} \mathrm{C}$. From their spectral responses, the work-of-adhesion value for bare toner particles (with no nanoparticle-coating) made of the same materials of the sample set utilized in the current study are predicted to be $W_{\mathrm{A}}=175$ $\mathrm{mJ} / \mathrm{m}^{2}$. This predicted $W_{A}$ value for bare toner particle is significantly higher than particles with 
$100 \%$ SAC, as previously observed and reported in [13]. Although the toner particles used in the current sample set and ones used in [13] are made of similar materials, the reported values (Table 1) differ possibly due to the difference in the particle mean diameters. The work-of-adhesion $\left(W_{\mathrm{A}}\right)$ of the microparticles is extracted from the acquired resonance frequencies $\left(f_{\mathrm{R}}\right)$ using a previously reported adhesion model [16]: $W_{A}=(56 / 45) \pi^{2} f_{R}^{2} r^{3} \rho$ where $r$ and $\rho$ are the rocking radius and the mass density of the particle, respectively. The work-of-adhesion $\left(W_{A}\right)$ and the surface energy $(\gamma)$ are related by $\gamma=W_{\mathrm{A}} / 2$. The decreasing trend observed for the rocking resonance frequencies correspond to the decrease in the work-of-adhesion (surface energy) for increasing temperature (Table 1). This trend is in general agreement with data reported in the literature [9-11]. The frequency resolution in the obtained spectral response plots is $0.2 \mathrm{kHz}$ (significantly lower than observed frequency shifts) contributes to an uncertainty of \pm 0.003 $\mathrm{mJ} / \mathrm{m}^{2}$ (work-of-adhesion), which is insignificant compared to the magnitudes of the reported work-of-adhesion values in Table 1 . It is thus concluded that the reported $W_{\mathrm{A}}$ values are unaffected by the experimental measurement uncertainties. The surface energy $(\gamma)$ for the surface-particle material pair $(M)$ is expressed by van der Waals and Guggenheim [19] as:

$$
\gamma\left(T_{s}, M\right)=\gamma^{o}(M)\left(1-\frac{T_{s}}{T_{c}(M)}\right)^{n(M)}
$$

where $T_{\mathrm{c}}(M)$ is the critical temperature of the material-pair $M$ and $\gamma^{\circ}(M)$ equals $\gamma$ at $T_{\mathrm{s}}=0 \mathrm{~K}$, and the exponent $n(M)$ is determined experimentally for the material-pair. Eq. 1 implies that the surface energy decreases with an increase in the surface temperature at thermal equilibrium. 
The average magnitude decrease in the work-of-adhesion for the reported temperature range (15$\left.30^{\circ} \mathrm{C}\right)$ is calculated to be $5.03 \mathrm{~mJ} / \mathrm{m}^{2}$. The percentage reductions in the work-of-adhesion $\left(\Delta W_{A}\right)$ values from $T_{s}$ of 15 to $30^{\circ} \mathrm{C}$ reported in Table 1 were categorized into two groups (5-30\%as Group I and $40-65 \%$ as Group II) and represented as a histogram in Fig. 4. The average and standard deviation (SD) of $\Delta W_{A}$ for Groups I $\left(W_{A}=22.3 \pm 11.52 \mathrm{~mJ} / \mathrm{m}^{2}\right.$ at $\left.T_{\mathrm{s}}=30^{\circ} \mathrm{C}\right)$ and II $\left(W_{A}\right.$ $=7.86 \pm 3.10 \mathrm{~mJ} / \mathrm{m}^{2}$ at $T_{\mathrm{s}}=30^{\circ} \mathrm{C}$ ) are also reported in Table 1 . The correlations between $W_{A}$ at $T_{\mathrm{s}}=30^{\circ} \mathrm{C}$ and $\Delta W_{A}$ are -0.1 for Group I and -0.72 for Group II. It is observed that this distribution of reduction values $\left(\Delta W_{\mathrm{A}}\right)$ appears to be bimodal. The distribution of the acquired rocking frequency values, corresponding work-of-adhesion values (Table 1) and the reduction in $\Delta W_{A}$ (Fig. 4) are attributed to the possible variability of nanoparticle dispersion on microparticle surfaces and, consequently, in the contact zones of the particle. The nanoparticle dispersion configurations in contact zones are categorized into three types, as depicted in Fig. 5, which is based on the surface morphology observed in the SEM image of the $100 \%$ SAC toner microparticle (Fig. 2(a)). On a close examination of the SEM image in Fig. 2(a), it is evident that the coating is non-uniform exposing the surface of base toner microparticle as depicted in the contact conformation Fig. 5(a) and 5(b). The contact nanoparticle distribution configuration in the contact zone shown in Fig. 5(a) is found to be improbable for the current sample set, as the estimated rocking resonance frequency for the bare toner particles (around $175 \mathrm{~mJ} / \mathrm{m}^{2}$ ) is considerably higher than the values reported in Table 1 for the sample set. It is therefore concluded that Groups I and II marked in Table 1 and Fig. 4 more likely to correspond to the coating particle configurations shown in Figs. 5(b) and 5(c). The adhesion reduction values in $\Delta W_{A}$ for Group II (marked bold in Table 1) were found predictable and well correlated with the extracted $W_{\mathrm{A}}$ values, whereas the reduction values of $\Delta W_{\mathrm{A}}$ in Group I were uncorrelated. Thus, it 
was concluded that the initial $W_{\mathrm{A}}$ values for particles in Group I at $15^{\circ} \mathrm{C}$ were a poor indicator of reduction in adhesion $\left(\Delta W_{\mathrm{A}}\right)$ at an increased temperature of $30^{\circ} \mathrm{C}$. However, for a microparticle in Group II, as suggested by the correlation value of -0.57 between the columns $W_{\mathrm{A}}$ at $T_{\mathrm{s}}=15^{\circ} \mathrm{C}$ and $\Delta W_{\mathrm{A}}$, the initial $W_{\mathrm{A}}$ values were found to be a good indicator of reduction level in adhesion, exhibiting a correlation of -0.72 with $\Delta W_{\mathrm{A}}$ at $T_{s}=30^{\circ} \mathrm{C}$. This observation in the correlations is attributed to the nanoparticle configurations depicted in Figs. 5(b) and 5(c). In Fig. 5(b), it is seen that the interfacial contact of silicon substrate and the toner microparticle is non-uniform, creating a particle distribution in the complex contact zone substantially different for each particle. If nanoparticles in the contact zone completely cover the bond zone nearly uniformly as in Fig 5(c), variations in the stiffness of the bonds for the particles is expected to be lower and more predictable compared to Group I. Finally, the low correlation value between the particle size and the reduction in $\Delta W_{\mathrm{A}}$ is reported in Table 1.

\section{Conclusions and Remarks}

In the current study, the surface energy dependence of the adhesion bond between a single nanoparticle coated toner microparticle and a silicon substrate on the substrate surface temperature is investigated using a non-contact/non-invasive experimental approach. From the reported experimental data and results, it is observed that the work-of-adhesion (surface energy) of nanoparticle coated toner microparticles with $100 \%$ SAC decrease with increasing the substrate surface temperature in the range of $15-30^{\circ} \mathrm{C}$. The effect of surface temperature on the reduction of work-of-adhesion values for the specified temperature range was found to be repeatable, and this experimentally observed reduction in the work-of-adhesion (surface energy) is in reasonable agreement with the published theoretical predictions for various materials. 
However, it is noted that the reductions for $100 \%$ SAC toner exhibit a bimodal distribution, which is attributed to the possible variability of nanoparticle dispersion on microparticle surfaces and in the adhesion contact zone. It is reported that the relative humidity (RH) could play a significant role in a microparticle rocking motion dynamics and its adhesion energy. In future studies, the temperature range is planned to be broadened to study the effects of higher temperatures $\left(>30^{\circ} \mathrm{C}\right)$ on the toner particles adhesion with various SAC levels. In addition, it is planned to conduct experiments at varying levels of local RH to study the effect of meniscus and capillary formation and condensation on microparticle adhesion. 


\section{Vitae}

Dr. Cetin Cetinkaya is a professor in the department of Mechanical and Aeronautical Engineering at Clarkson University. Dr. Cetinkaya's areas of research interests include solid mechanics, thermo-elastic wave propagation, transient finite element analysis and symbolic computing. He is the director of the Photo-Acoustics Research Laboratory at Clarkson University. Specific applications areas of the projects at the laboratory include laser ultrasonics, nondestructive evaluation of thin layered structures and testing/evaluation of small-scale objects. Educational Background:

Ph.D., University of Illinois - Urbana-Champaign

M.S., University of Illinois - Urbana-Champaign

B.Sc., Istanbul Technical University

Chaitanya Krishna Prasad Vallabh is currently a Ph. D. student under the supervision of Prof. Cetinkaya. He received his Master's degree in Mechanical Engineering in 2014 from Clarkson University under the supervision of Prof. Cetinkaya as well. His Bachelor of Science degree (2012) was in Aeronautical Engineering from Vignan's Institute of Technology and Aeronautical Engineering (affiliated to JNTU), Hyderabad, India.

James D. Stephens is currently a Ph. D. student under the supervision of Prof. Cetinkaya. He received his Bachelor of Science and Master's degree in Mechanical Engineering from Clarkson University. 
Dr. Grazyna Kmiecik-Lawrynowicz is a Principal Scientist at Xerox Corporation. She is responsible for designing new materials for xerographic applications. Her focus is on designing new chemical color toners prepared by nanotechnology approach called emulsion/aggregation process. She received her Master Degree in Chemistry and Chemical Engineering from Technical University in Warsaw Poland and her Ph.D. in Organic Chemistry from Rutgers - the State University of New Jersey. After postdoctoral work in Photochemistry at University of Toronto, she joined Xerox Research Center of Canada from which she was transferred to Xerox Supplies and Manufacturing in Webster. NY. Kmiecik-Lawrynowicz holds over 100 US patents.

Dr. Santokh S. Badesha is a Xerox Fellow and manager of open innovation for the Xerox Corporation. Badesha is responsible for leading inter-organizational efforts with both internal and external value chain partners, building academic and industrial partnerships, and providing critical technical support for product programs.

Badesha holds Bachelor and Master of Science degrees in chemistry from Punjab University, India. He received Ph.D. in organic chemistry from the Punjab Agricultural University and second Ph.D. in organic chemistry from the University of East Anglia, Norwich, U.K. He received an Honorary Doctorate of Science from Clarkson University. Badesha holds 199 U.S. Patents. 


\section{Acknowledgments}

The authors gratefully acknowledge financial support through a grant from the National Science Foundation (NSF) (Award Number: 1066877). Thanks are due to Xerox Corp. for providing samples of toner particles and stimulating discussions and guidance in handling of the microparticles. Partial funding in form of teaching assistantship was provided by Clarkson University. 


\section{REFERENCES}

1. Kmiecik-Lawrynowicz, G., New EA toners for high quality digital color printing, Int. Conf. Digital Production Printing and Industrial Applications 2 (2003) 211-213.

2. L.B. Schein, Applied physics. Recent progress and continuing puzzles in electrostatics, Science. 316 (2007) 1572-1573.

3. D.A. Hays, Adhesion of charged particles, J. Adhes. Sci. Technol. 9 (1995) 1063-1073.

4. D.A. Hays, J.C. Sheflin, Electrostatic adhesion of ion and triboelectric-charged particles, J. Electrostatics. 63 (2005) 687-692.

5. D. Rimai, D. Quesnel, L. DeMejo, M. Regan, Toner to photoconductor adhesion, J Imaging Sci Technol. 45 (2001) 179-186.

6. B. Gady, D. Quesnel, D. Rimai, S. Leone, P. Alexandrovich, Effects of silica additive concentration on toner adhesion, cohesion, transfer, and image quality, J Imaging Sci Technol. 43 (1999) 288-294.

7. C.K.P. Vallabh, A.S. Vahdat, C. Cetinkaya, Charge contribution to patch-charged microparticle adhesion, Appl. Phys. Lett. 105 (2014) 211905.

8. C.K.P. Vallabh, J.D. Stephens, G. Kmiecik-Lawrynowicz, S. Badesha, M. Sweeney, C. Cetinkaya, Predicting electrostatic charge on single microparticles, Powder Technol. 286 (2015) 684-696.

9. W. Luo, W. Hu, K. Su, F. Liu, The calculation of surface free energy based on embedded atom method for solid nickel, Appl. Surf. Sci. 265 (2013) 375-378.

10. T. Frolov, Y. Mishin, Temperature dependence of the surface free energy and surface stress: an atomistic calculation for $\mathrm{Cu}$ (110), Physical Review B. 79 (2009) 045430.

11. Q. Zhao, Y. Liu, E. Abel, Effect of temperature on the surface free energy of amorphous carbon films, J. Colloid Interface Sci. 280 (2004) 174-183.

12. D. Dowling, P. Kola, K. Donnelly, T. Kelly, K. Brumitt, L. Lloyd, R. Eloy, M. Therin, N. Weill, Evaluation of diamond-like carbon-coated orthopaedic implants, Diamond and Related Materials. 6 (1997) 390-393.

13. H. Zhang, W. Ding, K. Law, C. Cetinkaya, Adhesion properties of nanoparticle-coated emulsion aggregation toner, Powder Technol. 208 (2011) 582-589.

14. M.D.M. Peri, C. Cetinkaya, Non-contact microsphere-surface adhesion measurement via acoustic base excitations, J. Colloid Interface Sci. 288 (2005) 432-443. 
15. R. Baier, V. DePalma, D. Goupil, E. Cohen, Human platelet spreading on substrata of known surface chemistry, J. Biomed. Mater. Res. 19 (1985) 1157-1167.

16. Akseli, M. Miraskari, H. Zhang, W. Ding, C. Cetinkaya, Non-contact rolling bond stiffness characterization of polyvinylpyrrolidone (PVP) particles, J. Adhes. Sci. Technol. 25 (2011) 407-434.

17. C.K.P. Vallabh, J.D. Stephens, C. Cetinkaya, Effect of surface temperature on microparticle-surface adhesion, Appl. Phys. Lett. 107 (2015) 041607.

18. D. Schulze, Powders and Bulk Solids: Behavior, Characterization, Storage and Flow, Springer Science \& Business Media, 2007.

19. E.A. Guggenheim, The principle of corresponding states, J. Chem. Phys. 13 (1945) 253261. 
Figure 1: Instrumentation connectivity diagram of the experimental set-up for SAW-induced microparticle rocking and rolling (not-to-scale), the schematic of the SAW wave pulse and the close up image of the experimental are shown in the inset.

Figure 2: (a) SEM image of nanoparticle-coated (100\% SAC) toner microparticle on silicon substrate (side view at 10,000 $\times$ magnification) [13], (b) optical microscope image of a toner microparticle imaged at 100× magnification with a scale bar (Sample Set 01: P07 JL 29), and (c) microparticle map imaged at 5× magnification (Sample Set 01: JL 29)

Figure 3: Temporal and spectral responses of toner microparticles (a) P01 AG06 and (b) P04 AG06 for the temperature range of $15-30^{\circ} \mathrm{C}$.

Figure 4: Histogram representation of percentage reduction in the work-of-adhesion $\left(\Delta W_{A}\right)$ values (Table 1) from the surface temperature of $15^{\circ} \mathrm{C}$ to $30^{\circ} \mathrm{C}$. The bars indicate the average and standard deviation (SD) of $\Delta W_{A}$ for Groups I and II.

Figure 5: Schematics of three possible contact conformations between fully coated (SAC of 100\%) toner and silicon substrate: $(a)$ microparticle in direct contact with the substrate; $(b)$ partial contact of microparticle silica nanoparticles with the substrate, blocking the rocking motion of the microparticle; and (c) the layer of silica nanoparticles in contact with the substrate. 
Table 1: The acquired rocking resonance frequencies $\left(f_{R}\right)$ and the corresponding estimated workof-adhesion $\left(W_{A}\right)$ values for the particles in the date set SS01 at four levels of surface temperature $\left(T_{s}=15^{\circ} \mathrm{C}, 20^{\circ} \mathrm{C}, 25^{\circ} \mathrm{C}\right.$ and $\left.30^{\circ} \mathrm{C}\right)$. The statistics for particles in Group I (G I) and Group II (G II indicated in bold) are also shown. SD and Corr stand for standard deviation of the corresponding group and the correlation coefficient between its respective column and the column entitled $\% \Delta \mathrm{W}_{\mathrm{A}}$ (the percentage reduction in $\mathrm{W}_{\mathrm{A}}$ from $15^{\circ} \mathrm{C}$ to $30^{\circ} \mathrm{C}$ ). 


\section{Surface Temperature $\left(T_{s}\right)$}

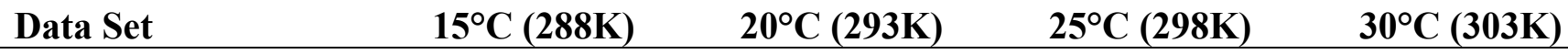

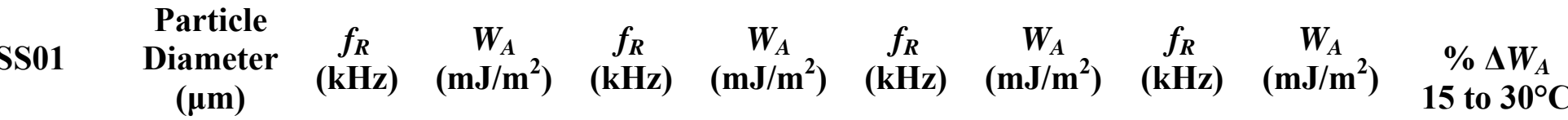

\begin{tabular}{|c|c|c|c|c|c|c|c|c|c|c|}
\hline P01 MR16 & 12 & 149.5 & 14.82 & 140.4 & 13.07 & 134.3 & 11.96 & 128.2 & 10.9 & 26.45 \\
\hline P02 MR16 & 14 & 119 & 18.33 & 91.5 & 10.84 & 86.9 & 9.78 & 83.9 & 9.11 & $\mathbf{5 0 . 3 0}$ \\
\hline P02 JL02 & 17 & 157.2 & 46.59 & 155.6 & 45.65 & 154.1 & 44.7 & 149.5 & 42.14 & 9.55 \\
\hline P05 JL02 & 20 & 111.4 & 38.1 & 105.3 & 34.04 & 103.8 & 33.08 & 96.13 & 28.37 & 25.54 \\
\hline P01 JL29 & 13 & 70.57 & 13.21 & 57.22 & 8.68 & 55.31 & 8.11 & 53.41 & 7.56 & $\mathbf{4 2 . 7 7}$ \\
\hline P07 JL29 & 13 & 76.29 & 19.63 & 72.48 & 17.71 & 70.57 & 16.79 & 68.66 & 15.9 & 19.00 \\
\hline P01 AG06 & 12 & 84.69 & 37.16 & 80.87 & 33.88 & 78.58 & 31.99 & 77.82 & 31.37 & 15.58 \\
\hline P02 AG06 & 15 & 74.77 & 23.55 & 74.01 & 23.07 & 64.09 & 17.3 & 62.56 & 16.48 & 30.02 \\
\hline P04 AG06 & 18 & 74.2 & 49.29 & 71.33 & 45.55 & 69.43 & 43.16 & 67.9 & 41.28 & 16.25 \\
\hline P06 AG06 & 12 & 71.72 & 32.34 & 67.52 & 28.66 & 66.18 & 27.54 & 64.28 & 25.98 & 19.67 \\
\hline P03 SP08 & 14 & 51.52 & 19.71 & 48.45 & 17.7 & 47.3 & 16.87 & 46.92 & 16.6 & 15.78 \\
\hline P04 SP08 & 18 & 99.37 & 30.31 & 98.61 & 29.85 & 97.85 & 29.39 & 96.32 & 28.48 & 6.04 \\
\hline P02 SP17 & 15 & 53.6 & 21.67 & 46.35 & 16.2 & 43.87 & 14.51 & 38.91 & 11.41 & 47.35 \\
\hline P03 SP17 & 14 & 78.01 & 45.9 & 77.82 & 45.67 & 76.87 & 44.57 & 71.72 & 38.79 & 15.49 \\
\hline P01 SP29 & 13 & 33.76 & 3.84 & 33.38 & 3.75 & 32.81 & 3.63 & 31.47 & 3.34 & 13.02 \\
\hline P05 SP29 & 19 & 56.08 & 33.11 & 53.98 & 30.68 & 51.88 & 28.34 & 50.54 & 26.89 & 18.79 \\
\hline P02 OC08 & 11 & 61.61 & 7.75 & 59.70 & 7.28 & 47.68 & 4.64 & 38.15 & 2.97 & 61.68 \\
\hline P07 OC08 & 13 & 82.40 & 22.90 & 75.91 & 19.43 & 74.96 & 18.95 & 72.86 & 17.90 & 21.83 \\
\hline P09 OC08 & 13 & 90.03 & 27.33 & 86.59 & 25.29 & 85.45 & 24.62 & 84.88 & 24.30 & 11.09 \\
\hline P02 OC15 & 14 & 45.59 & 8.75 & 44.82 & 8.46 & 43.3 & 7.89 & 42.34 & 7.55 & 13.71 \\
\hline P03 OC15 & 15 & 49.02 & 15.11 & 48.26 & 14.64 & 37.96 & 9.06 & 36.24 & 8.25 & 45.40 \\
\hline P04 OC15 & 16 & 56.84 & 16.74 & 56.08 & 16.29 & 55.69 & 16.06 & 55.12 & 15.74 & 5.97 \\
\hline P05 OC15 & 12 & 63.71 & 10.76 & 63.32 & 10.63 & 62.94 & 10.50 & 60.65 & 9.75 & 9.39 \\
\hline Average & 14.48 & 78.73 & 24.21 & 74.33 & 22.04 & 71.38 & 20.58 & 68.63 & 19.18 & 23.51 \\
\hline $\mathrm{SD}$ & 2.41 & 30.58 & 12.67 & 28.84 & 12.44 & 29.06 & 12.40 & 28.35 & 11.63 & 15.20 \\
\hline G I Avg & 14.72 & 80.95 & 26.71 & 78.14 & 24.97 & 76.12 & 23.74 & 73.77 & 22.32 & 16.29 \\
\hline G I SD & 2.56 & 32.47 & 13.42 & 31.26 & 12.83 & 30.76 & 12.51 & 29.39 & 11.52 & 6.79 \\
\hline Corr GI & -0.101 & & 0.048 & & 0.001 & & -0.061 & & -0.1 & \\
\hline G II Avg & 13.6 & 70.76 & 15.21 & 60.61 & 11.53 & 54.34 & 9.22 & 50.12 & 7.86 & 49.50 \\
\hline G II SD & 1.50 & 28.19 & 5.27 & 18.18 & 3.81 & 19.26 & 3.55 & 20.08 & 3.10 & 7.34 \\
\hline Corr GII & -0.733 & & -0.571 & & -0.499 & & -0.561 & & -0.72 & \\
\hline Corr SS01 & -0.229 & & -0.340 & & -0.413 & & -0.469 & & -0.509 & \\
\hline
\end{tabular}




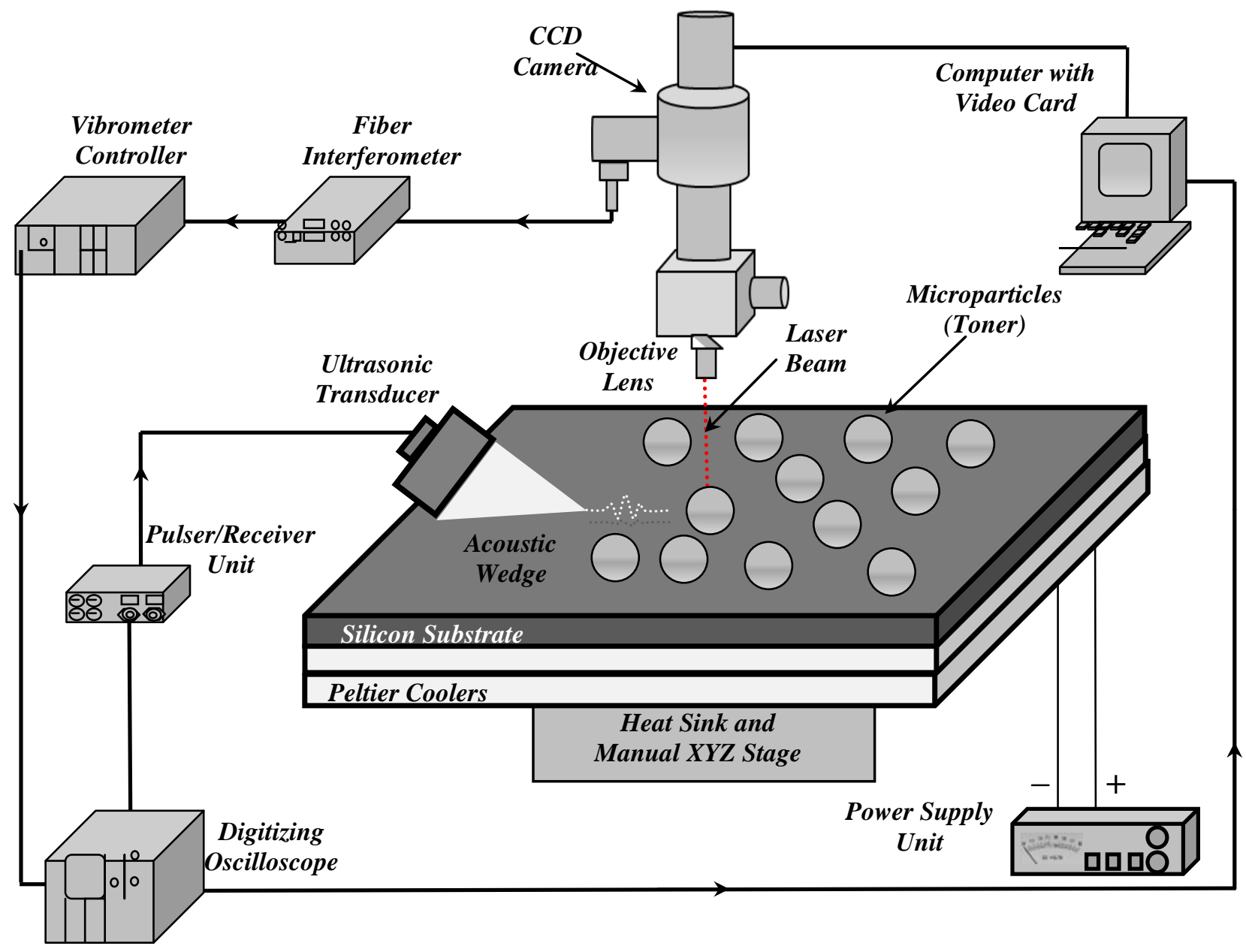




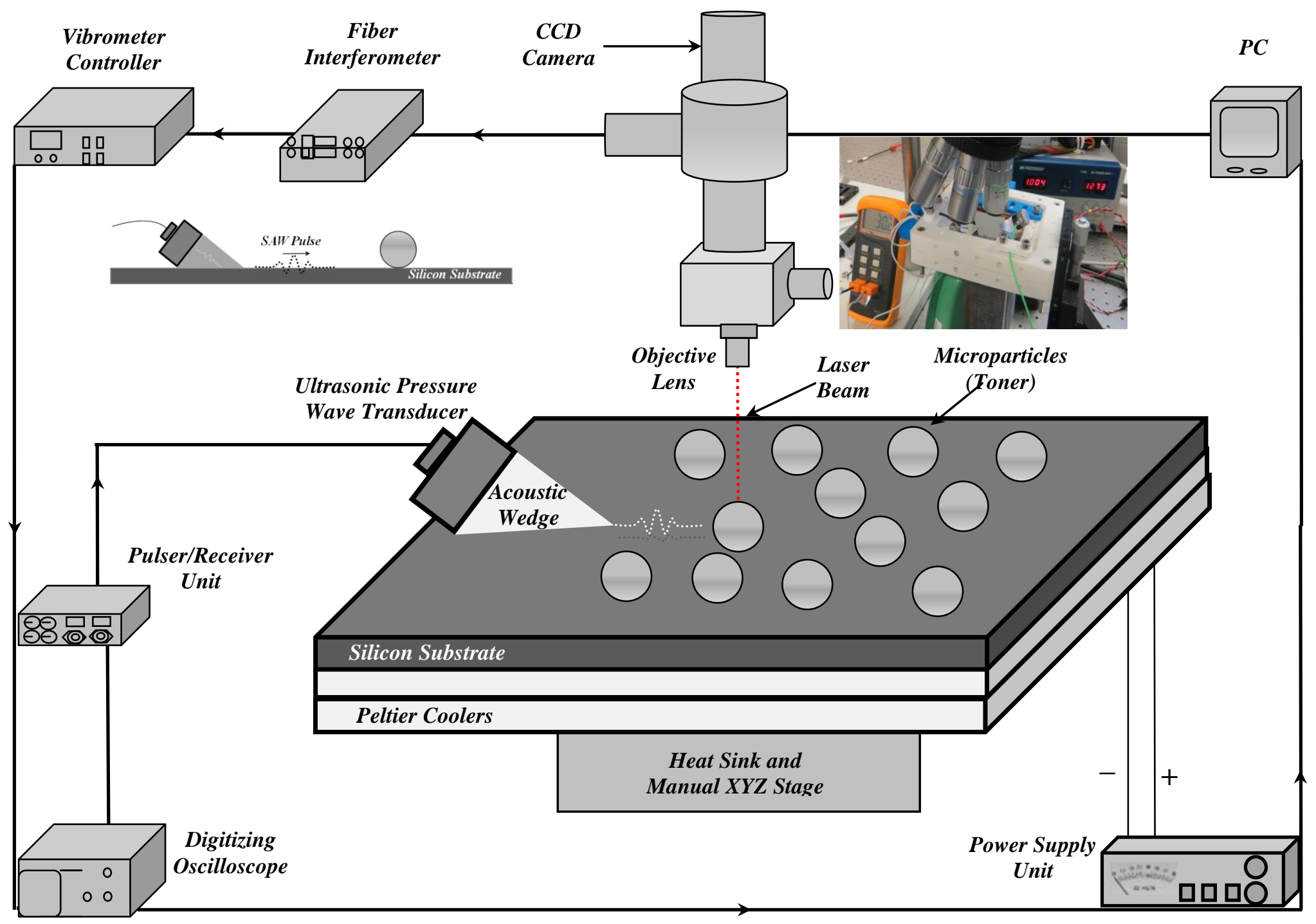




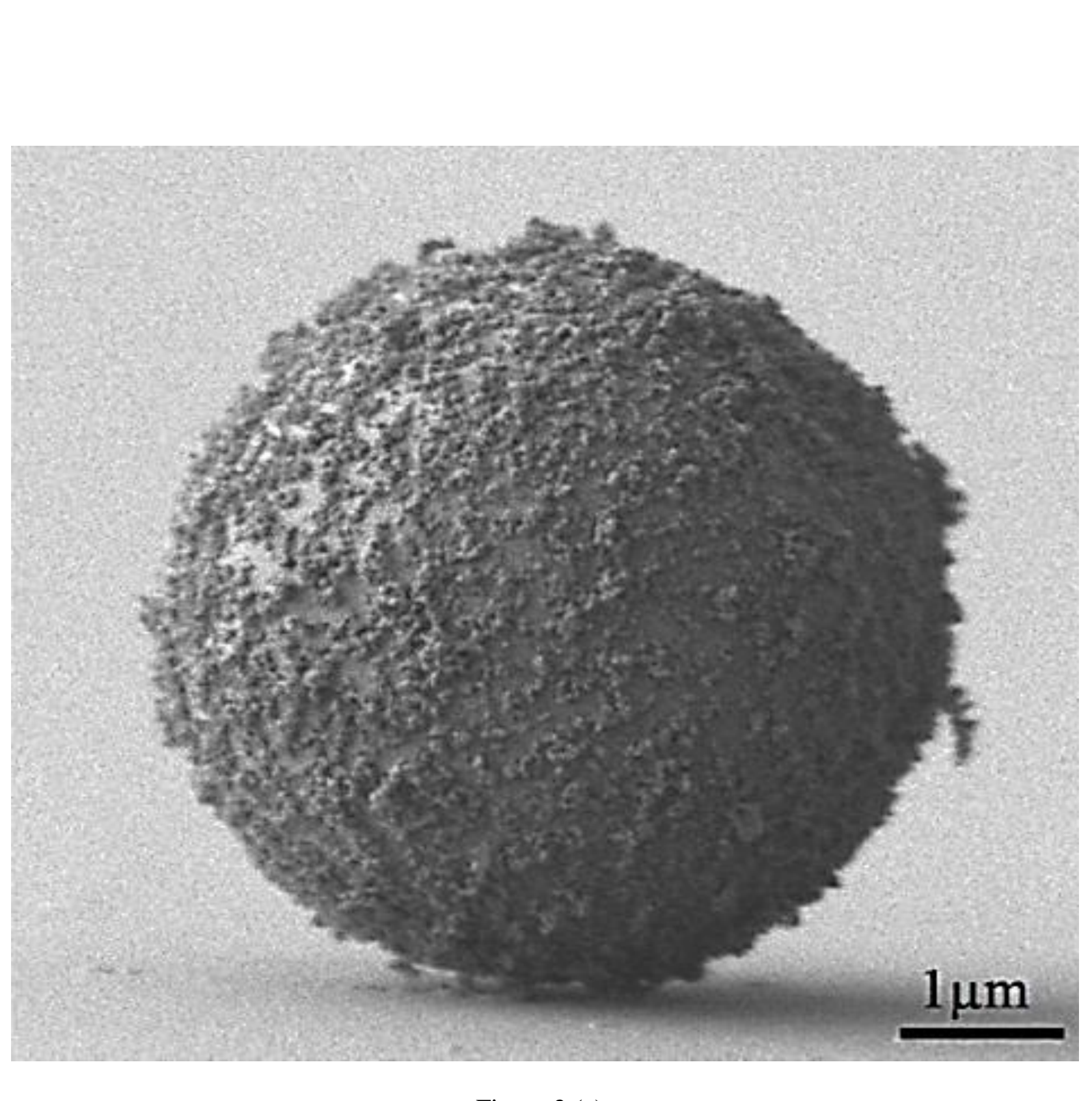

Figure 2 (a)

Fig.2.a

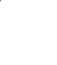

a




.



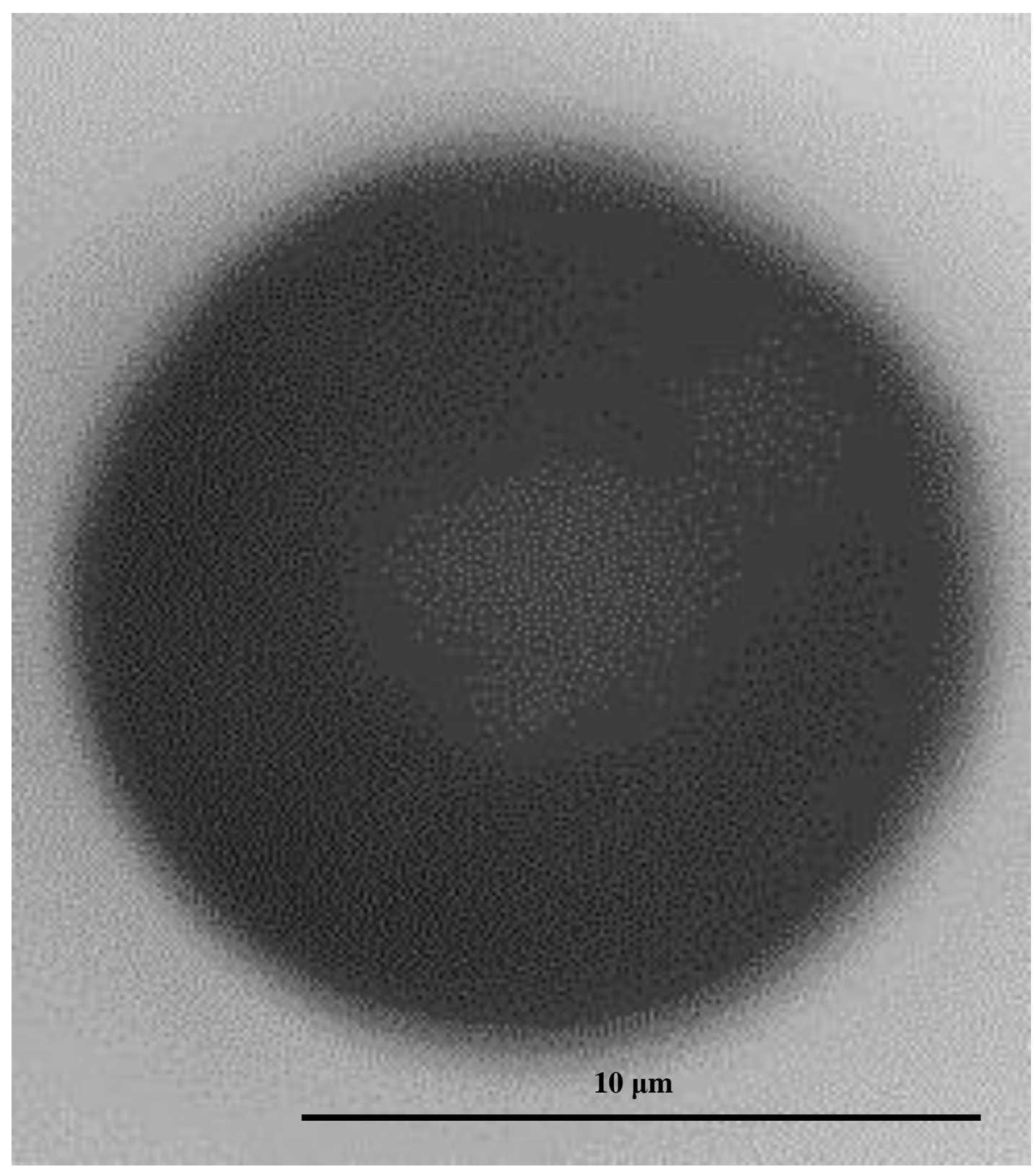

Figure 2 (b)

Fig.2.b

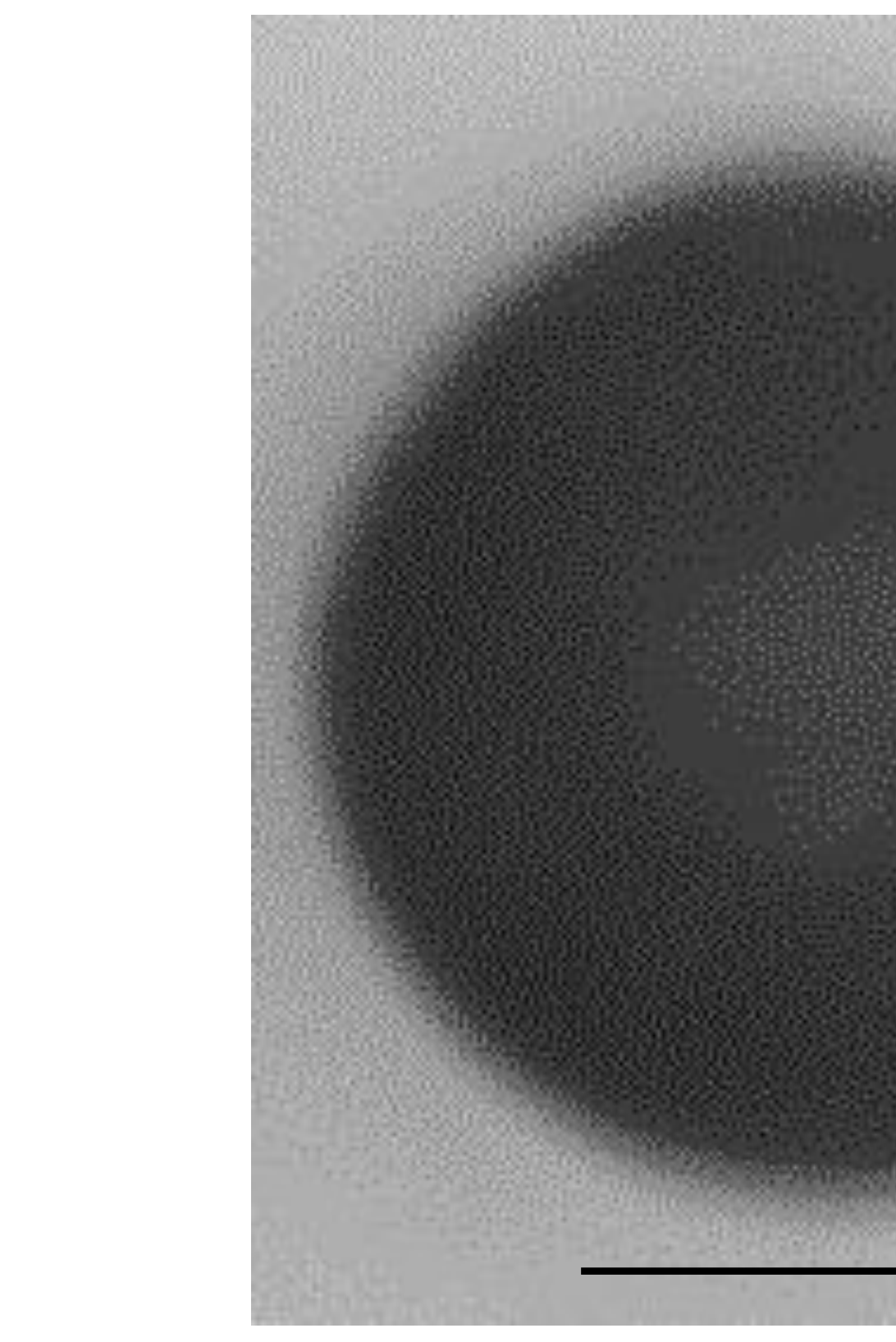

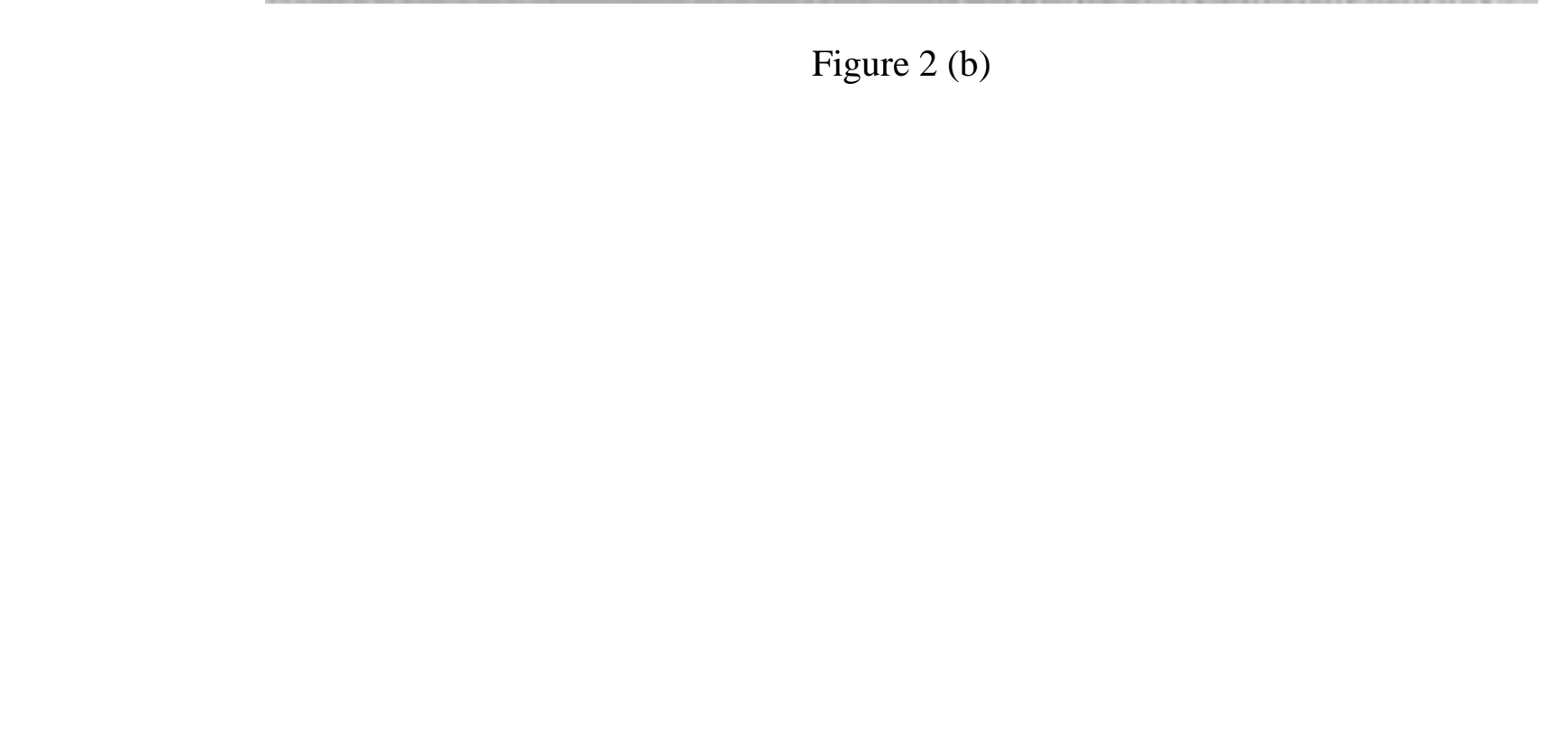

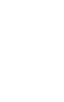

\author{
(
}

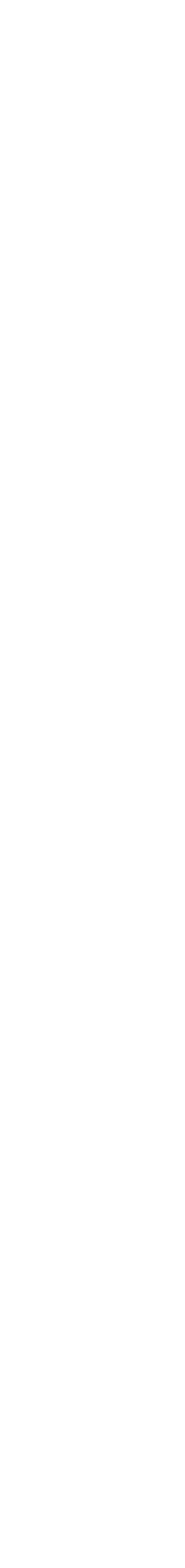

(2) (



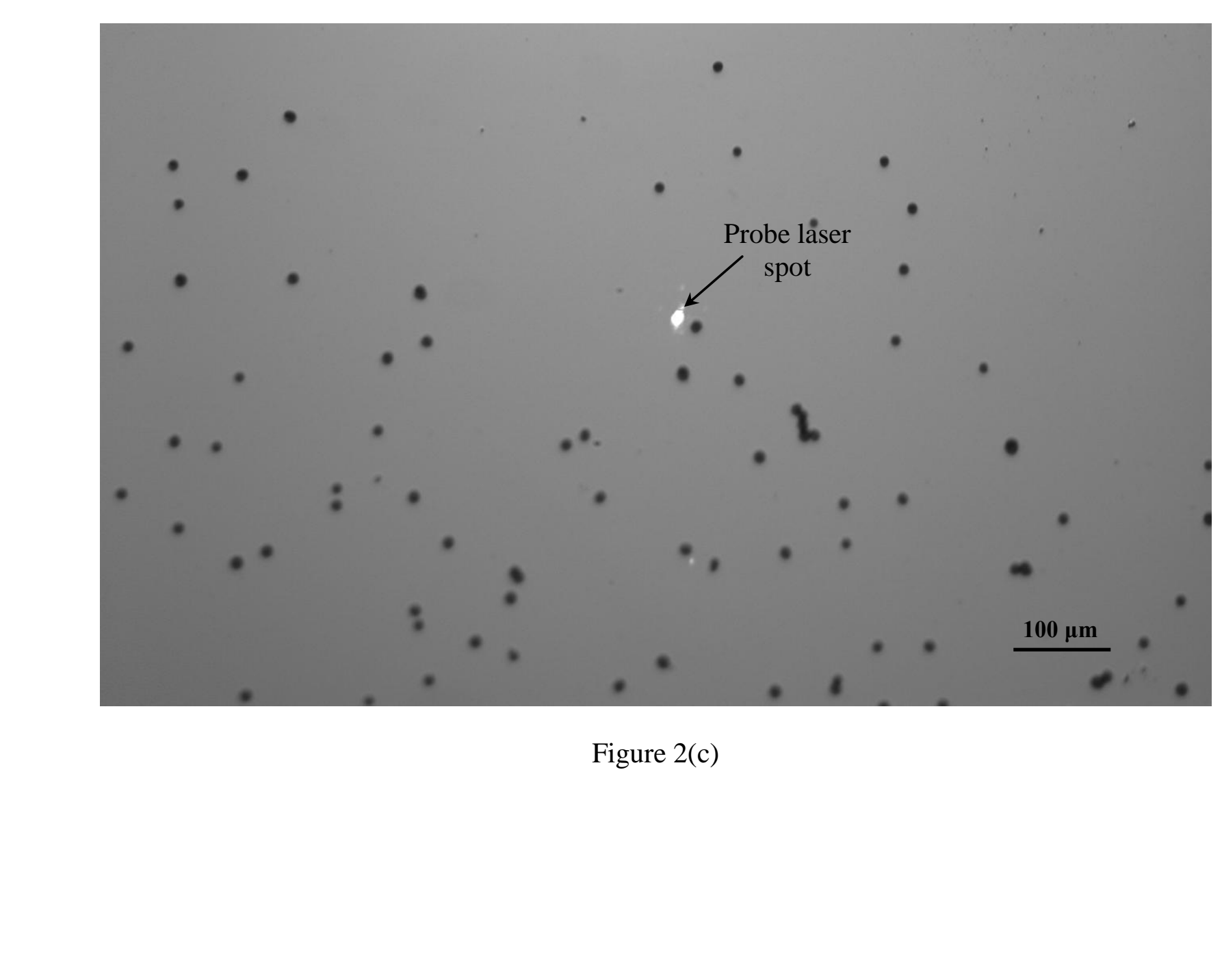

Figure 2(c)

Fig.2 c

Figure 2(c)

.
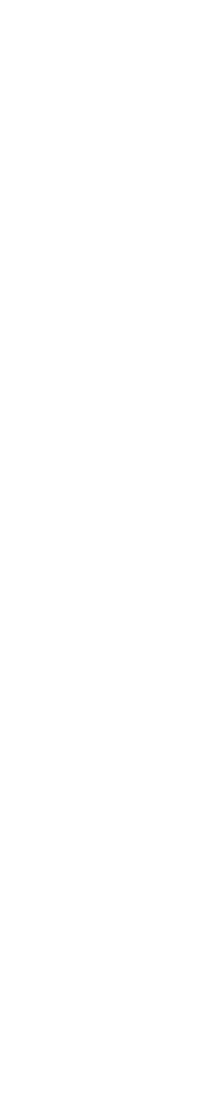

Fin
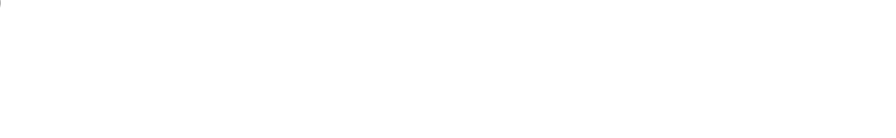

.

Fig.3.a
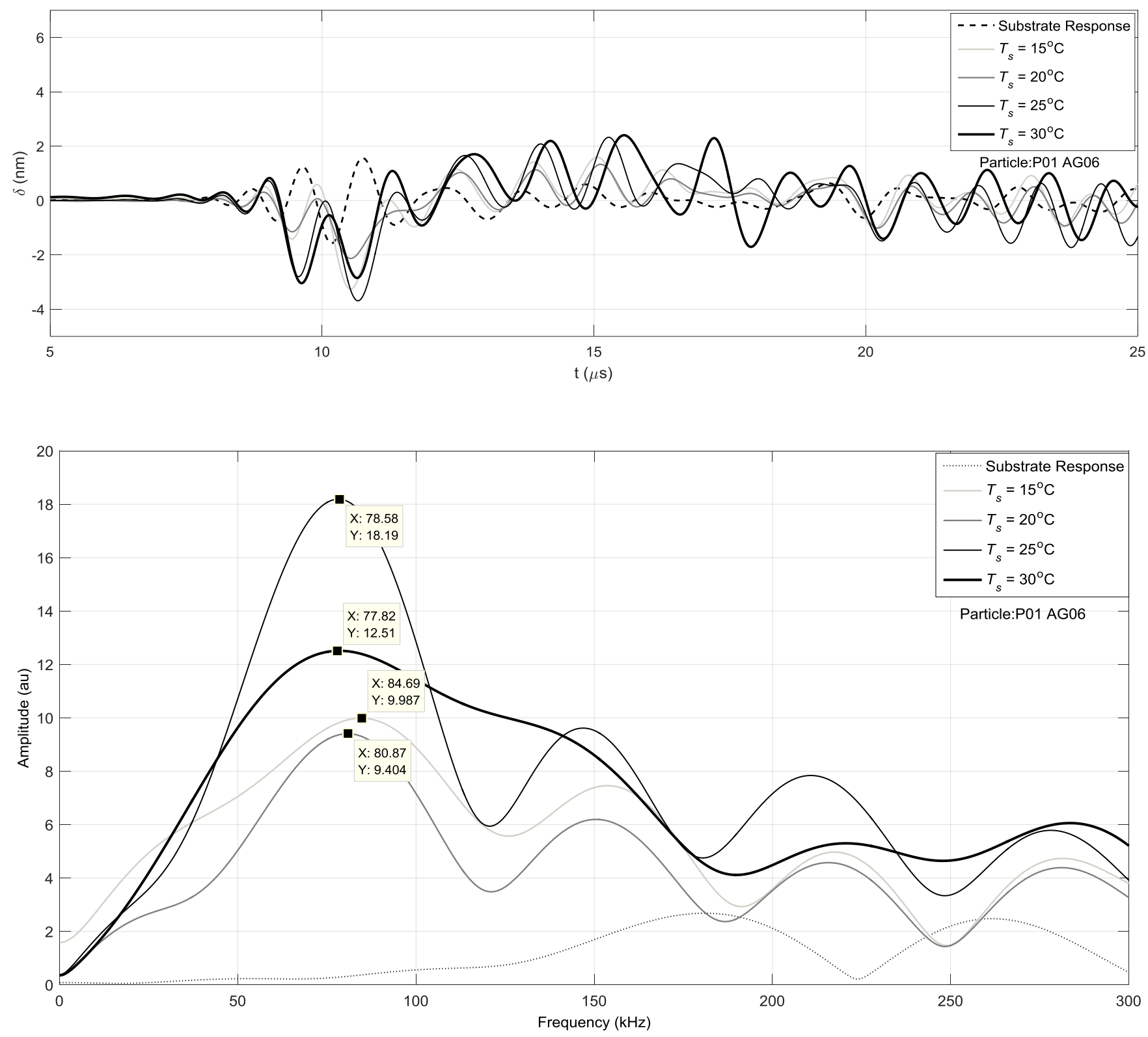

Figure 3 (a) 



Figure 3 (b) 


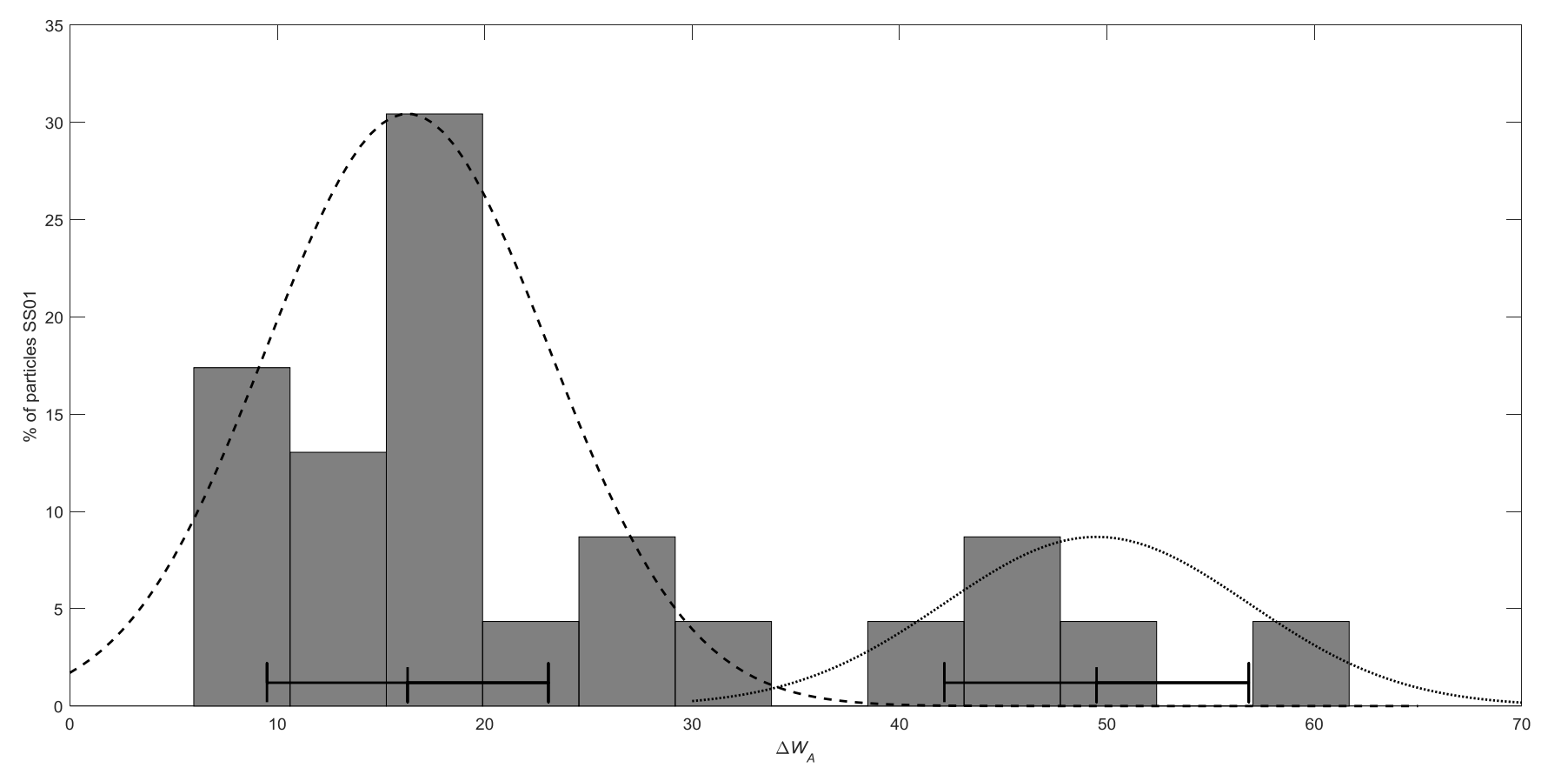

Fig.4 
Fig.5.a

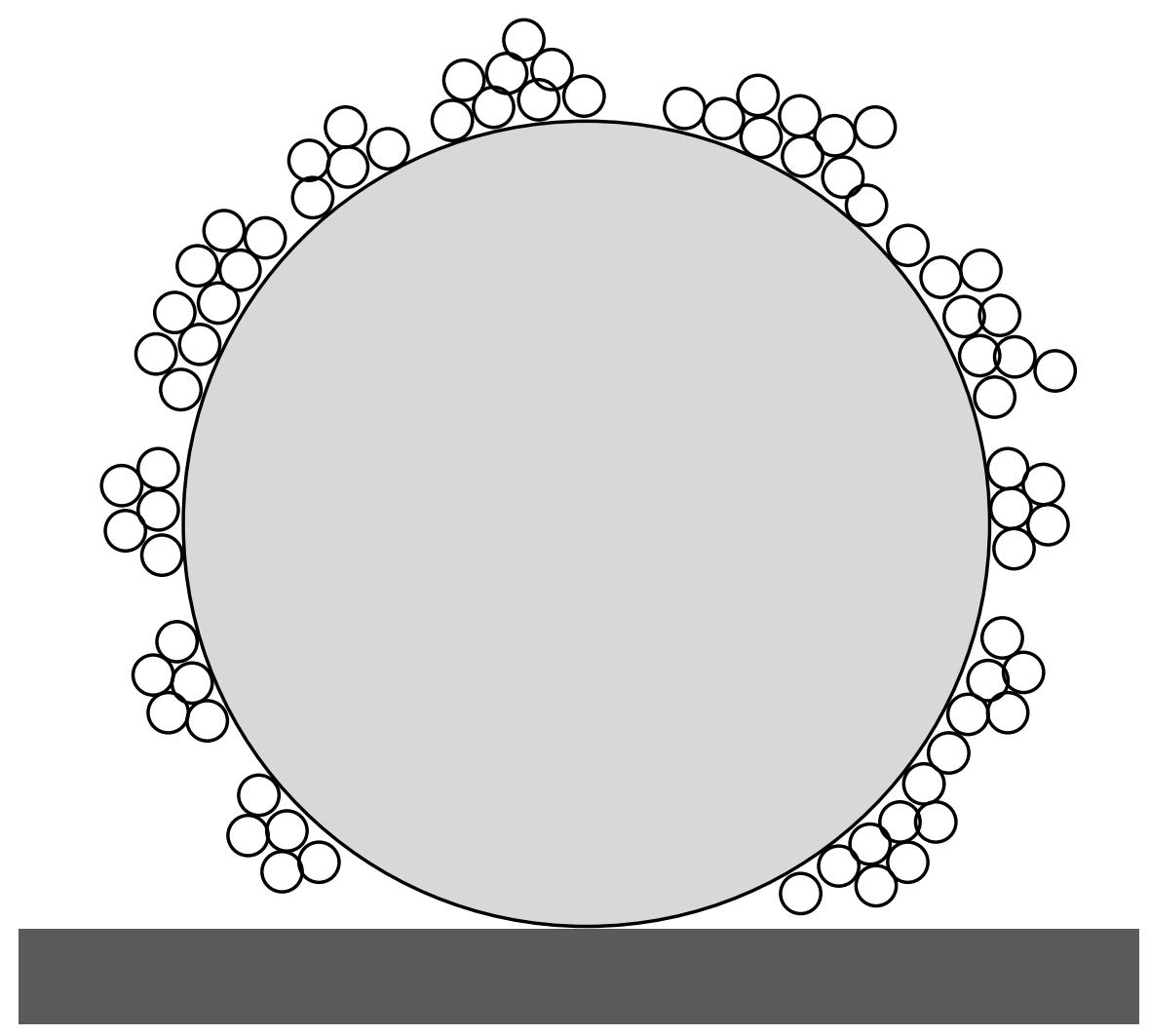

Figure 5(a) 


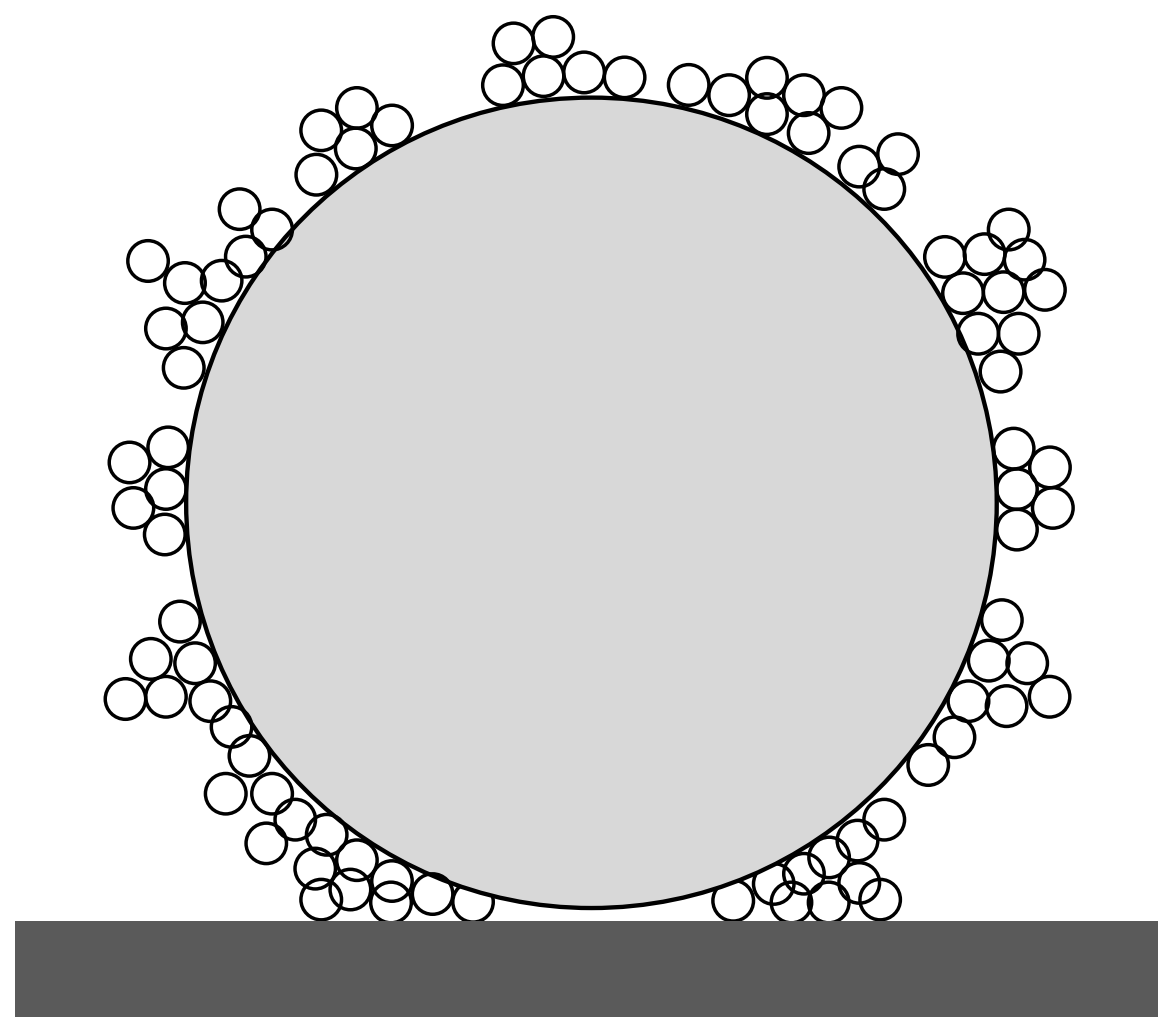

Figure 5(b)

Fig.5.b 
Fig.5.c

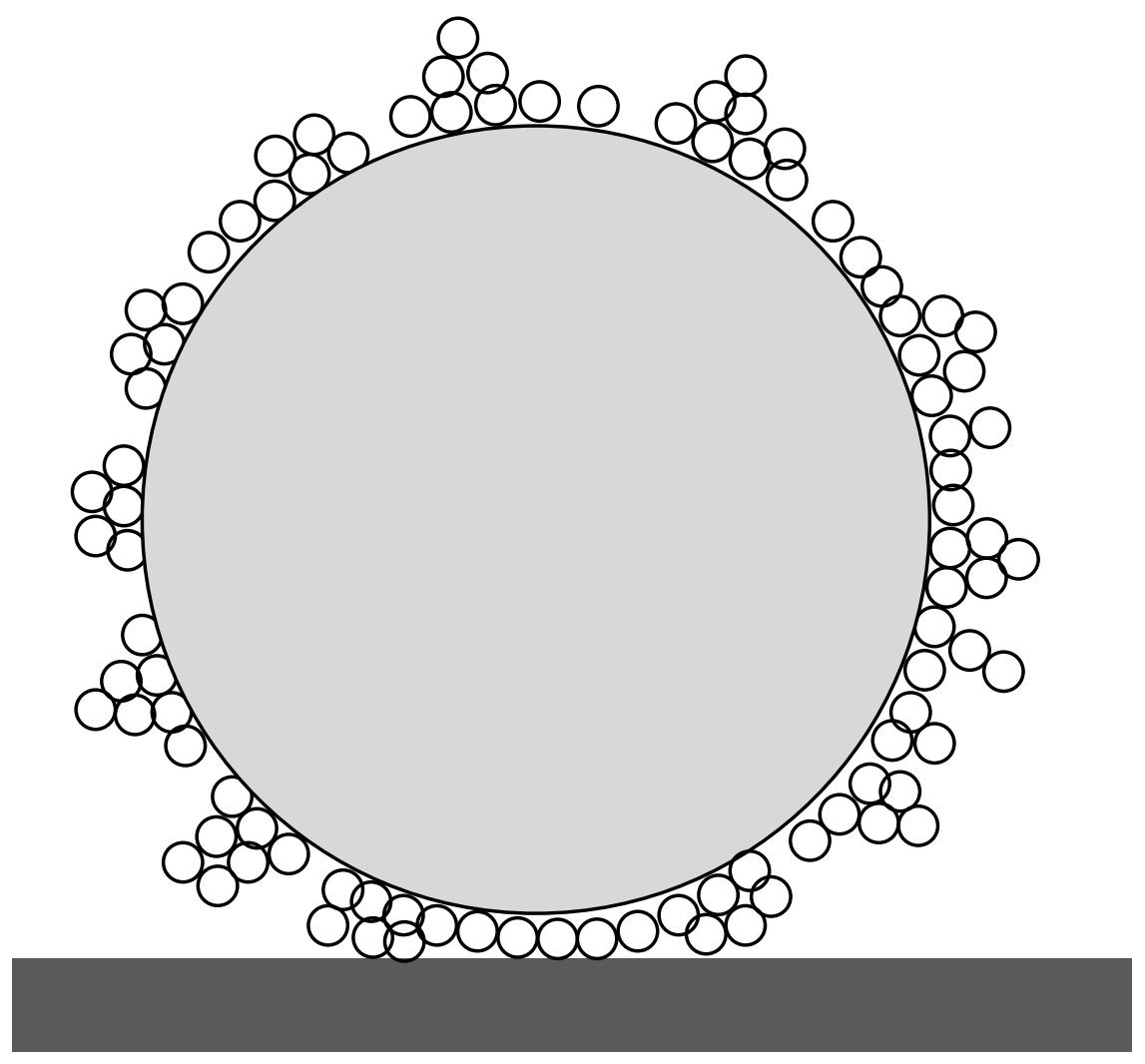

Figure 5(c) 